\title{
A Case of Linear Scleroderma Involving Cerebellum with Vertigo
}

\author{
Eui Je Choi, Dong Won Lee, Chul Won Park and Seung Hwan Lee \\ Department of Otolaryngology-Head and Neck Surgery, College of Medicine, Hanyang University, Seoul, Korea
}

\author{
Received June 1, 2012 \\ Revised August 2, 2012 \\ Accepted August 31, 2012
}

\section{Address for correspondence \\ Seung Hwan Lee, MD \\ Department of Otolaryngology- \\ Head and Neck Surgery, \\ College of Medicine, \\ Hanyang University, \\ 222 Wangsimni-ro, Seongdong-gu, \\ Seoul 133-791, Korea \\ Tel +82-31-560-2363 \\ Fax +82-31-566-4884 \\ E-mail shleemd@hanyang.ac.kr}

Linear scleroderma is a kind of disease that can cause complication of the central nervous system. Sometimes, ipsilateral intracerebral or white matter lesions in the brain magnetic resonance imaging are noted. Nystagmus is important for the differential diagnosis of dizziness. Positional nystagmus was classified into regular direction nystagmus, direction changing positional nystagmus (DCPN) and irregular nystagmus by their character. DCPN is defined as a nystagmus that changes its direction with different head and body positions, and it can be the sign of lesion in the central vestibular system. Recently, we experienced a 17-year-old woman who had a scalp linear scleroderma, treated for about 10 years and showed DCPN induced by positional nystagmus test. We report a case of linear scleroderma accompanied by cerebellar lesion with a brief review of literature.

Korean J Audiol 2012;16:87-90

\section{Introduction}

The directional change of nystagmus, which is definitely identified by a positional nystagmus test, can be related to central vertigo. In 1920, Barany defined positional nystagmus as a nystagmus that occurs with keeping the head in a specific position, which is a major sign of lesion in the central vestibular system. Most of the direction changing positional nystagmus (DCPN) is known to occur in benign paroxysmal positional vertigo, ${ }^{1)}$ but also the central lesion, such as cerebellar degeneration, brainstem infarction and multiple sclerosis, etc. ${ }^{2)}$

Linear scleroderma is a kind of disease that can cause complication of the central nervous system. Sometimes, ipsilateral intracerebral or white matter lesions in brain magnetic resonance imaging (MRI) are noted. There is no report that the cerebellar calcification by linear scleroderma is the reason of DCPN, until nowadays. Recently, we experienced a case of 17-year-old female patient who had been treated for linear scleroderma for ten years, accompanied by vertigo and cerebellar lesion. DCPN was noted in the positional nystagmus test and brain image studies showed multiple calcified lesions in the cerebellum. We report this patient with a brief review of the literature.

\section{Case Report}

A 17-year-old female patient visited our hospital for continuous spinning sense, which had been occurring for the past three days. The dizziness was continuous at rest, and aggravated with the moving head, accompanied by nausea and vomiting. The patient complained of left hearing loss that began approximately 1 year prior. There were no other otologicsymptoms, such as otalgia, ear fullness or otorrhea, etc. She had been taking Methotrexate (MTX) for linear scleroderma of the scalp since 2001, and had a history of undergoing resection and primary closure for progressive depression of the left forehead skin in 2010. Otoscopic examination of the ear showed that the external auditory canals and tympanic membranes in both ears were normal. Pure-tone audiometry showed sensorineural hearing loss of about $53 \mathrm{~dB}$ HL on the left side and normal hearing on the right side (Fig. 1). Tympanogram showed type A on both sides and stapedial reflex test was normal. In videonystagmography, spontaneous nystagmus toward the right side was observed (Fig. 2). The patient was admitted under suspicion for vestibular neuronitis and routine blood tests, electrocardiogram, and chest X-ray after admission were normal.

In positional nystagmus test, on the second hospital day, 
DCPN was observed. The nystagmus to the right side ( $25 \mathrm{deg} / \mathrm{s}$ ) first appeared by rolling the head to the right in supine position, but when rolling the head to left, after about 58 seconds of right beating nystagmus ( $25 \mathrm{deg} / \mathrm{s})$, the direction of nystagmuswas changed to the left side (20 deg/s), without changing the head position (Fig. 3). Saccade test, pursuit test and optokinetic test presented normal ranges, andbithermal caloric test showed the left canal paresis of 19\% (Fig. 4). The neurologic and cerebral function exams were unremarkable. In cerebellar function tests, finger-to-nose test and heel-to-shin test were normal, but she showed instability in tandem gait test. Videonystagmography was performed again, and inhibition by visual fixation was not presented. With suspicion of central lesion, brain MRI and computed tomography were carried out. Multiple calcified lesions at the left cerebellum were observed. Compared with brain MRI in 2004, progressed lesions of the cerebellum were observed (Fig. 5). The cerebospinal fluid (CSF) study and eosinophil count study undergone for suspecting parasite infection were normal.

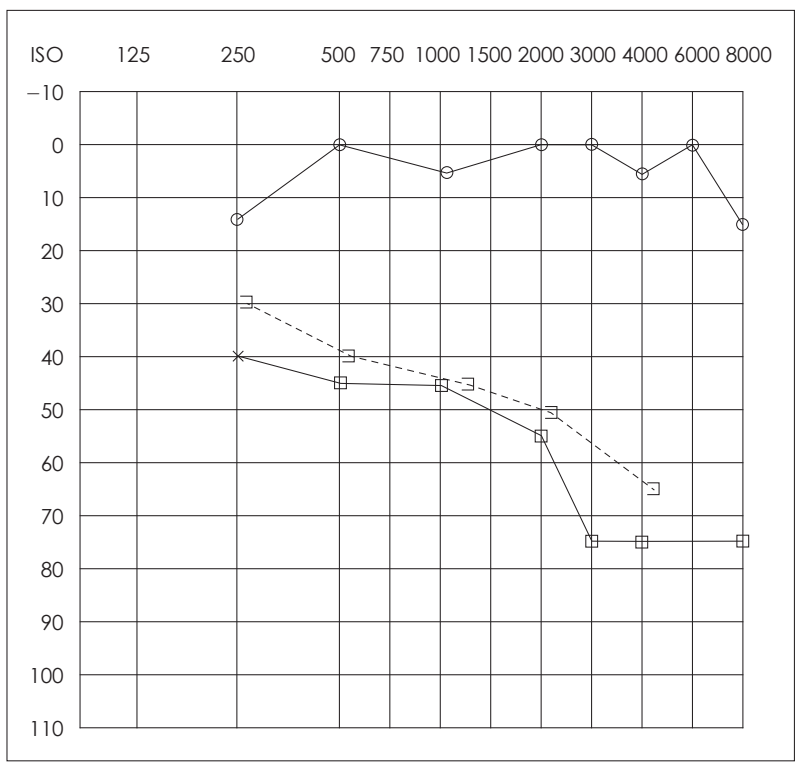

Fig. 1. Pure tone audiometry. Right hearing was normal and sensorineural hearing loss was noted at left side, about $53 \mathrm{~dB} H \mathrm{HL}$ in air conduction.
The patient was given a prescription for administration of antiemeticagent and antivertigo medication. Sixth day of admission, the patient was discharged because dizziness was improved. After two weeks, she still had the symptoms of dizziness, but it became mild and spontaneous nystagmus was not observed. At present, conservative treatment and observation is maintained.

\section{Discussion}

The elements that comprise nystagmus are duration, direction, regularity, and etc. A lot of efforts have gone into making a differential diagnosis with finding the cause of dizziness after analyzing them. According to Lin, et al. ${ }^{2}$ and Baloh and Honrubia $^{3)}$ DCPN does not always mean peripheral vestibular disease and also not localize the lesion in the peripheral vestibular pathway. But they reported that most of DCPN was observed when the lesion was in the peripheral vestibular system.

Lin, et al. ${ }^{2)}$ classified the causes of DCPN into central nervous system disease and peripheral vestibular system disease. For example, the central nervous system diseases are brainstem infarction, cerebellar degeneration, multiple sclerosis, Arnold-Chiari malformation, brain tumor, and etc. and peripheral vestibular disease includes sensorineural hearing loss with the canal paresis, Meniere's disease, chronic otitis media, acoustic tumor, and etc. Since 1985, McClure ${ }^{4)}$ reported for the first time that canalolithiasis or cupulolithiais of lateral semicircular canal was the most common cause of DCPN, the link between DCPN and peripheral vestibular disease increased. But there was no report that linear scleroderma could be the cause of DCPN as this case.

Linear scleroderma is one of the autoimmune diseases and causes localized hardening of the skin on the arm, leg or forehead, which affects four times more women than men. In $83 \%$ of patients, the symptoms are expressed gradually before 25 years old. It has activating period of $2-3$ years, which is progressed by trauma in $23 \%$. It can also affect the muscle or bone tissue under the skin lesion. Especially, in case of causing linear band atrophy in the frontal area, we called it frontal linear

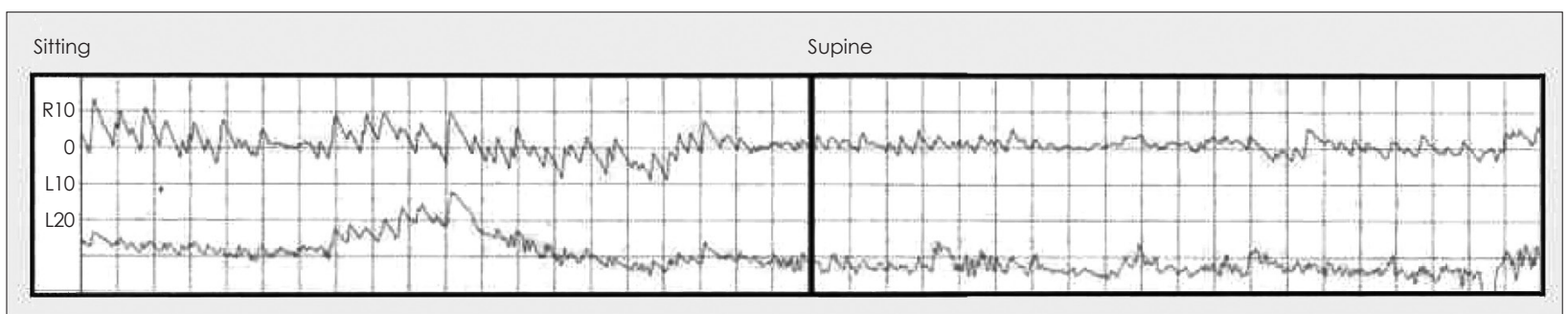

Fig. 2. Spontaneous nystagmus to right (6.9 deg/s) under video nystagmography on the 1st OPD visit day (upper line: vertical nystagmus, lower line: horizontal nystagmus). OPD: outpatient department. 
Roll test-Head right

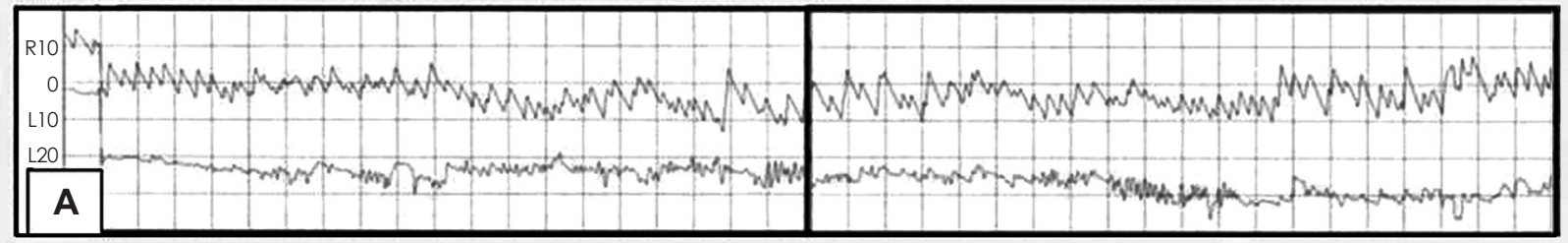

Roll test-Head left (1-40s)

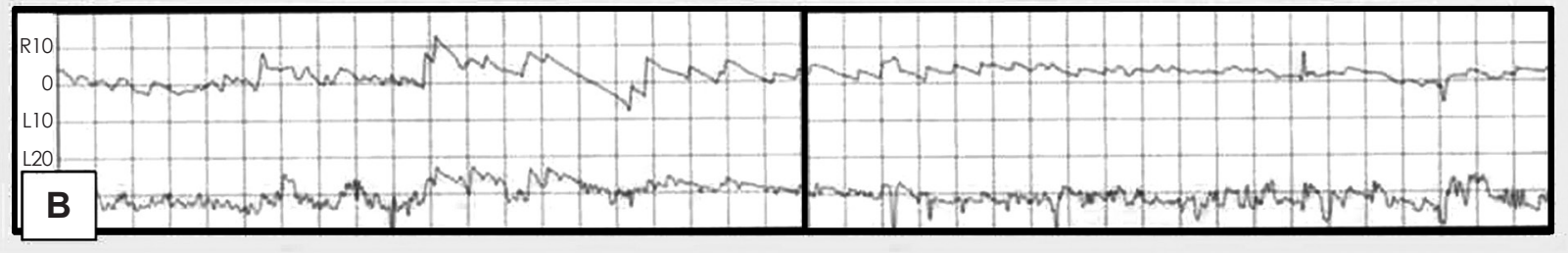

Roll test-Head left (41-80 s)

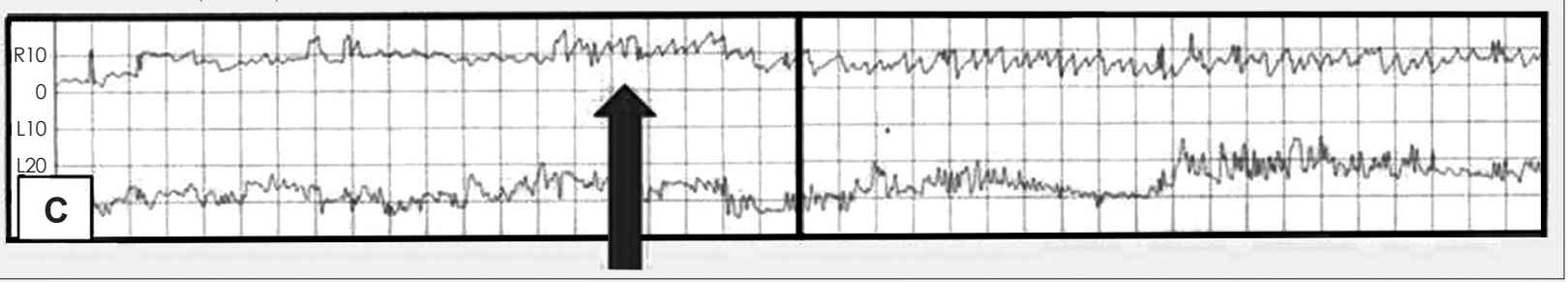

Fig. 3. Videonystagmography on the second hospital day. Right beating nystagmus ( $25 \mathrm{deg} / \mathrm{s})$ was shown when rolling head to right (A). When rolling head to left, right beating nystagmus ( $25 \mathrm{deg} / \mathrm{s})$ was shown during 1-58 seconds $(\mathrm{B})$, but the direction of nystagmus was changed to left side (20 deg/s) at the point of arrow, after 58 soconds (C) (upper line: vertical nystagmus, lower line: horizontal nystagmus).

Fig. 4. Caloric test. Caloric test shows canal paresis $(19 \%)$ of left side. SPV: slow phase peak velocity.
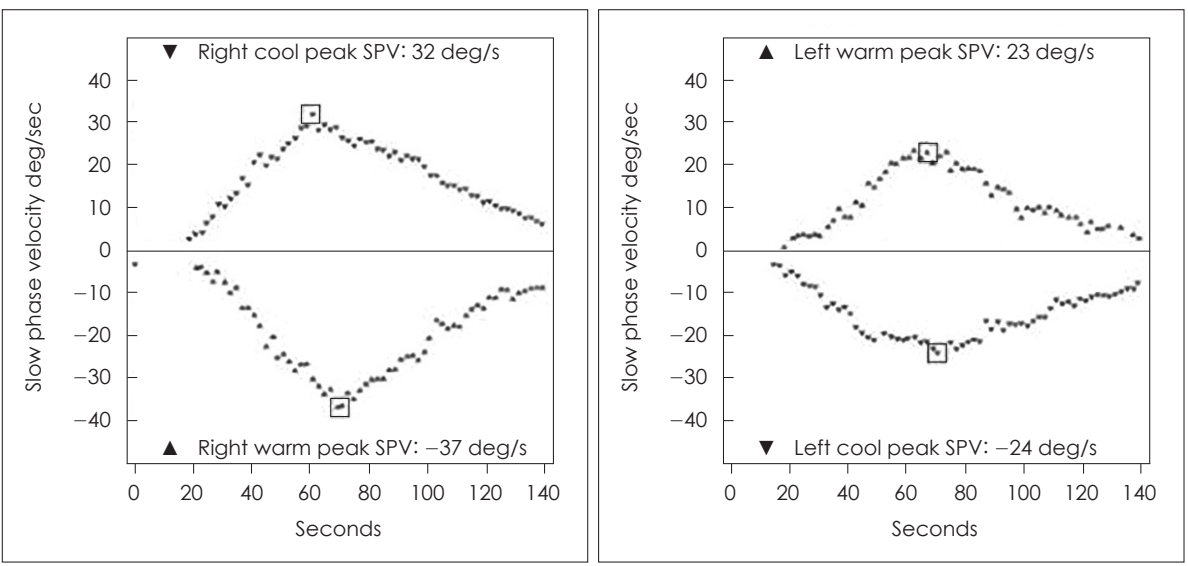

scleroderma or "en coup de sabre" (LSCS). ${ }^{5)}$ Current treatments, including MTX, Methylprednisolone IV pulse therapy and low dose ultraviolet A1 (UVA1) phototherapy have been proven to be effective and the patient in this case has maintained such a treatment for about ten years.

The pathogenesis of LSCS is unknown, but associated with neurological complications. Though it is uncommon, ipsilateral brain lesion can be observed and epileptic seizures or complications of the eye, unilateral facial atrophic changes involving the skin, a variety of neurological symptoms can appear. ${ }^{5)}$
But white matter lesions in brain MRI are observed sometimes without neurological symptoms.

This case of linear scleroderma patient was being treated with MTX in the dermatologic department for ten years. In 2004, partial calcifications of the left cerebellum were observed in brain MRI she had undergone for evaluation of the brain lesions associated with LSCS at the dermatologic department; but no neurological symptoms showed up. As the calcified lesions gradually progressed, dizziness could occur by decreasing vestibular function and inhibition signals through vestib- 

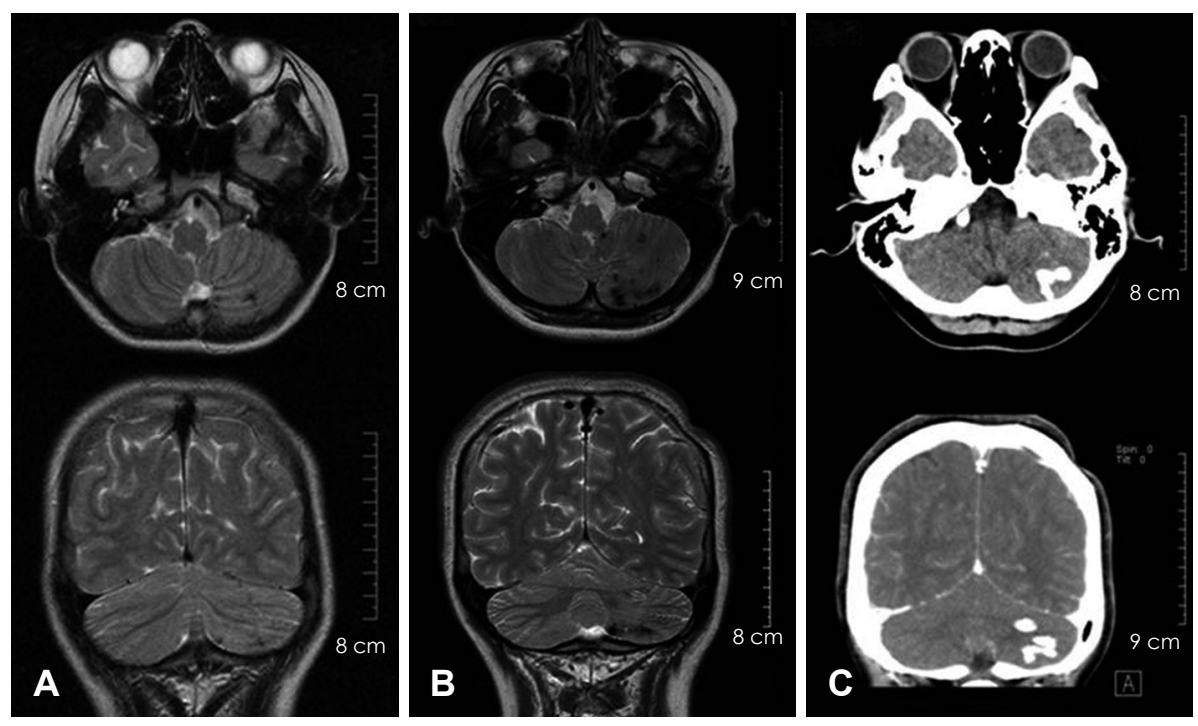

Fig. 5. Axial scans of brain magnetic resonance image and computed tomography. A: On the T2 weighted image of 2004, several signal void lesions were found on posterior lobe of left cerebellar hemisphere. B: The area of signal void lesions seemed to be enlarged on 2011. C: There were white calcified parenchymal lesions on the same site of left cerebellum on brain CT with enhancement.

ulo-cerebellar pathway, due to adjacent vascular swelling or spasm. Diseases with calcification in the cerebellum include parasitic infections, such as Sparganosis or Paragonimiasis, Fahr disease ${ }^{6}$ caused by idiopathic or familial reason, and secondary calcification caused by a reduction of parathyroid hormone. We ruled these out by parasite examination and blood tests.

We started conservative treatment under suspicion of unilateral vestibular function disorder, such as vestibular neuronitis due to acute vertigo and spontaneous nystagmus of the patient. But in positional nystagmus test on the second hospital day, DCPN was observed and the result of bithermal caloric test presented normal ranges, the left canal paresis of $19 \%$. Because visual fixation was also not applied, brain MRI and computed tomography were carried out for differential diagnosis. We considered the possibility that the vertigo and DCPN in this patient could be related to the enlarged cerebellar calcified lesions, compared to the images in 2004.

After conservative treatment for administration of antiemetic agent and antivertigo medication, dizziness was improved and the patient was discharged. After two weeks, she still had the symptoms of dizziness, but it became mild and observation is maintained.

In case of acute vertigo attack, we should evaluate the dizziness in the viewpoint of both peripheral and central vestibular lesions. Also, close examinations about the characters of nystagmus and dizziness is essential. Especially, a confirmation whether central vestibular lesion exists or not is helpful for the diagnosis and treatment when DCPN is observed in relatively younger patient.

\section{REFERENCES}

1) Ichijo H. Persistent direction-changing geotropic positional nystagmus. Eur Arch Otorhinolaryngol 2012;269:747-51.

2) Lin J, Elidan J, Baloh RW, Honrubia V. Direction-changing positional nystagmus: incidence and meaning. Am J Otolaryngol 1986;7:30610.

3) Baloh RW, Honrubia V. The history of the dizzy patient. In: Eggers $\mathrm{SD}$, Zee DS, editors. Clinical Neurophysiology of the Vestibular System. 3rd ed. New York: Oxford university press;2001. p.111-4.

4) McClure JA. Horizontal canal BPV. J Otolaryngol 1985;14:30-5.

5) Holland KE, Steffes B, Nocton JJ, Schwabe MJ, Jacobson RD, Drolet BA. Linear scleroderma en coup de sabre with associated neurologic abnormalities. Pediatrics 2006;117:e132-6.

6) Ellison D, Love S, Chimelli L, Harding BN, Lowe J, Vinters HV. Calcium disturbances and Fahr's disease. In: Jennifer S, Kelly W, editors. Neuropathology: a Reference Text of CNS Pathology. 2nd ed. London: Mosby;2004. p.428-9. 